Received: 2017.01 .21

Accepted: 2017.03.31

Published: 2017.09 .26
Authors' Contribution: Study Design A Data Collection B Statistical Analysis C Data Interpretation D Manuscript Preparation E Literature Search F Funds Collection G

\title{
Association of Serum Galectin-3 with the Acute Exacerbation of Chronic Obstructive Pulmonary Disease
}

ABCDEF Wei Feng

$B C D$ Xiaojuan $\mathrm{Wu}$

ACDF Shaojun Li

ABCD Cui Zhai

BCDE Jian Wang

BCDF Wenhua Shi

ACDFG Manxiang Li
Corresponding Author: Source of support:

Manxiang Li, e-mail: manxiangli@hotmail.com

This work was supported by the National Science Foundation of China (No.81330002)

Department of Respiratory and Critical Care Medicine, The First Affiliated Hospital of Xi'an Jiaotong University, Xi'an, Shaanxi, P.R. China

Background: Acute exacerbation of chronic obstructive pulmonary disease (AECOPD) aggravates the overall severity in COPD patients, resulting in severe morbidity and mortality. However, there are no objective biomarkers currently available to predict the development of AECOPD. Several studies have indicated that galectin-3 (Gal-3) is involved in diseases characterized by excessive inflammatory response and fibrosis. The objective of this study was to examine the dynamic changes of Gal-3 in acute exacerbation and convalescence phases of COPD.

Material/Methods: Serum levels of Gal-3, high sensitivity C-reactive protein (hsCRP), and prohormone of brain natriuretic peptide (pro-BNP) were determined using multiplex enzyme-linked immunosorbent assay kits. Serum levels of Gal-3 in 44 patients with COPD were further analyzed and correlated with the parameters of lung function and the biomarkers of systemic inflammation.

Results: The mean level of serum Gal-3 was significantly higher in acute exacerbation of COPD compared with the level in COPD convalescence phase (32.10 \pm 9.83 versus $29.02 \pm 8.68 \mathrm{ng} / \mathrm{mL}, p<0.01)$. Serum levels of Gal-3 positively correlated with hsCRP ( $r=0.354, p=0.018$ for total patients) and pro-BNP ( $r=0.319, p=0.035$ for total patients) in AECOPD. In addition, the level of Gal-3 was the highest in the current smoker group, and the lowest in the never-smoker group in either the acute exacerbation phase $(33.91 \pm 3.55$ versus $29.12 \pm 11.73 \mathrm{ng} / \mathrm{mL}, p=0.036)$ or the convalescence phase $(30.94 \pm 3.40$ versus $27.76 \pm 9.68 \mathrm{ng} / \mathrm{mL}, p=0.045)$ of COPD.

Conclusions: Our results indicated that serum Gal-3 is increased in AECOPD patients, which is also positively associated with systemic inflammation and smoking in patients with COPD, suggesting that Gal-3 might be a valuable biomarker for AECOPD.

MeSH Keywords: Biological Markers • Galectin $3 \bullet$ Inflammation • Pulmonary Disease, Chronic Obstructive

Full-text PDF: https://www.medscimonit.com/abstract/index/idArt/903472

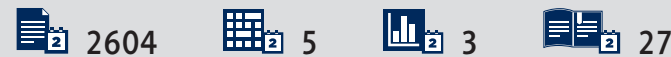




\section{Background}

Chronic obstructive pulmonary disease (COPD) is a debilitating disease characterized by persistent progressive airflow limitation resulting from enhanced chronic inflammation in the airways in response to noxious particles or gases [1]. Apart from local inflammation in the lungs, COPD patients often show increased systemic inflammation, which is associated with the pathogenesis of comorbidities present in COPD, especially for those who are experiencing exacerbations [2]. Exacerbations and comorbidities aggravate the overall disease-associated morbidity and mortality in COPD patients. However, to date, there are few measurable prognostic serum biomarkers predicting exacerbation risk in COPD. Thus, identification of novel biomarkers for early detection and evaluation of prognosis for AECOPD is of considerable clinical importance.

Galectin-3 (Gal-3), a $\beta$-galactoside-binding lectin, is expressed by different kinds of cells, mainly activated macrophages, and produced by tissues such as lung, heart, and liver. Evidence indicates that Gal-3 is an important pro-inflammatory mediator and has been extensively investigated in various inflammatory conditions, including heart diseases, cancers, and systemic sclerosis. It is also involved in profibrotic responses, inducing proliferation and transformation of fibroblasts, and mediating collagen deposition into the extracellular matrix [3,4]. Gal-3 can stimulate the production of some cytokines and chemokines via CD98 through interactions with macrophages [5]. In heart failure, elevated levels of Gal-3 have been positively correlated with prohormone of brain natriuretic peptide (pro$B N P)$, indicating that Gal-3 may be involved in the process of myocardial remodeling and right ventricle dysfunction $[6,7]$.

Recently, it has been shown that Gal-3 is an emerging prognostic indicator in pulmonary diseases. In the lung, expression of Gal-3 in epithelial is increased in non-small cell lung cancer compared with healthy controls [8], while in a rat asthma model, allergen-induced airway inflammation was inhibited by gene therapy targeted to Gal-3, including the expression of IL-5 and epithelial mucous metaplasia [9]. Secretion of Gal3 in circulating systems was also elevated in a mouse model of radiation-induced lung fibrosis [10]. In addition, Gal-3 has been shown to be a binding partner of $\beta$-catenin and Axin to play a central role in Wnt/B-catenin signaling that is involved in upregulating extracellular matrix (ECM) constituents and matrix metalloproteinases (MMP) $[11,12]$. In summary, these data indicate that Gal-3 has a potent regulatory role in inflammation and fibrogenesis.

In COPD, recent findings demonstrated that elevated Gal3 levels in blood and lung tissues were correlated with increased epithelial injuries and airflow obstruction in severe COPD patients [13]; this suggests that it may have potential as a prognostic biomarker and may be a promising therapeutic target for COPD. However, to our knowledge, Gal-3 levels in COPD patients during the acute exacerbation and convalescence have not yet been investigated. Therefore, we conducted this prospective cohort study to examine serum Gal-3 levels in acute exacerbation and convalescence phases of COPD and compared these levels to established biomarkers, including pro-BNP and hsCRP, to explore the utility of Gal-3 as a potential biomarker for AECOPD.

\section{Material and Methods}

\section{Patients}

A total of 106 patients with AECOPD were admitted to the Department of Respiratory Medicine of The First Affiliated Hospital of Medical College, Xi'an Jiaotong University (Xi'an, Shaanxi, China) from October 2014 to March 2016. Sixty-two AECOPD patients with coronary artery disease, diabetes, autoimmune diseases, chronic kidney disease, and malignancy which potentially affected Gal-3 levels, were excluded. Fortyfour AECOPD patients met the inclusion criteria. The present prospective cohort study was approved by the Research Committee of Human Investigation of Medical College of Xi'an Jiaotong University and each patient provided informed consent.

\section{Inclusion and exclusion criteria}

Patients with AECOPD were defined by the following criteria: 1) diagnosed as COPD according to the Global Initiative for Chronic Obstructive Lung Disease (GOLD) guidelines [1], who had a history of chronic respiratory symptoms, such as cough and sputum with or without breathlessness, had a post-bronchodilator forced expiratory volume in $1 \mathrm{~s}\left(\mathrm{FEV}_{1}\right) /$ forced vital capacity (FVC) ratio of less than $0.7 ; 2$ ) a worsening of respiratory symptoms (baseline dyspnea, cough, and/or sputum production) beyond normal day-to-day variations, leading to a change in medication [1]. Convalescence of COPD was determined by the following criteria: symptoms of patient with COPD return to the pre-exacerbation level variability, physician carefully assessed the patient and confirmed that the individual was medically stable enough to leave the hospital, but patient was not unstable and prone to acute exacerbation again [14].

Exclusion criteria were as follows: 1) any chronic cardiopulmonary disease other than COPD including asthma and pneumonia; 2) received oral or intravenous corticosteroids or any other anti-inflammatory drugs in the preceding four weeks, given the possibility that anti-inflammatory drugs may be able to hamper the elevation of Gal-3 and thus confound the results; 3) with any condition such as coronary artery disease, diabetes, autoimmune diseases, chronic kidney disease, and malignancy 
which could affect Gal-3 levels; 4) an inability to give written informed consent or cooperate with the study investigators.

\section{Measurements of clinical parameters}

Detailed clinical and demographic data were collected at admission. We also obtained the hospital days, smoking history (shown by number of pack-years=number of cigarettes smoked per day/20x number of years smoked, one pack has 20 cigarettes), course of disease and exacerbation frequency in previous three years where obtained from patient declarations. Laboratory measurements, including total leukocyte counts, neutrophils\%, serum hsCRP, pro-BNP, and arterial blood gas analyses were performed. Chest CT was performed for each patient to assess the severity of emphysema, and to exclude pneumonia and other pulmonary diseases.

\section{Pulmonary function tests}

Each patient underwent pulmonary function tests according to the American Thoracic Society guidelines using a Pulmonary Function Test System (MasterScreen, Erich Jaeger GmbH, Germany) by the same technician. Reversibility assessment was performed in all COPD patients by inhaling a short-acting $\beta 2$ agonist equivalent to $200 \mu \mathrm{g}$ salbutamol. For all inpatients with AECOPD who met the inclusion criteria, spirometry was conducted at 10 to 14 days after the onset of exacerbation when the patients were stable enough to perform the spirometric testing.

\section{Blood sampling and processing}

Peripheral blood was collected at the following two time points: within 24 hours of hospital admission; before discharge from hospital after treatment for 10 to 14 days in hospital. Blood was drawn and stored by the same researcher in the same department. Blood samples were collected aseptically in coagulated tubes and stored at $4^{\circ} \mathrm{C}$. Samples were centrifuged at $1,000 \mathrm{rpm}$ at $4^{\circ} \mathrm{C}$ for 15 minutes, and then serum was collected and stored at $-80^{\circ} \mathrm{C}$ until analysis.

\section{Measurements of Gal-3, hsCRP, and pro-BNP}

Serum Gal-3 levels were measured using commercial enzymelinked immunosorbent assay (ELISA) kits (R\&D Systems, Inc., Minneapolis, MN, USA). The lower detectable limit of the Gal-3 using these kits was $0.1 \mathrm{ng} / \mathrm{mL}$. Each sample was run in duplicate and compared with a standard curve. The mean concentration was determined for each sample. HsCRP and pro-BNP were measured immediately at a clinical laboratory once blood serum was acquired.
Table 1. The basic characteristics of the study subjects.

\begin{tabular}{|cc|}
\hline \multicolumn{1}{c}{ Parameter } & Value \\
\hline $\mathrm{N}$ & 44 \\
\hline Age (years) & $69.18 \pm 9.36$ \\
\hline Sex (Male/Female) & $34 / 10$ \\
\hline BMI $\left(\mathrm{kg} / \mathrm{m}^{2}\right)$ & $22.32 \pm 3.16$ \\
\hline Never/current/ex-smokers & $16 / 9 / 19$ \\
\hline Smoking history (pack/years) & $21.4 \pm 14.4$ \\
\hline Course of disease (years) & $27.82 \pm 9.13$ \\
\hline Hospitalization time (days) & $12(10-14)$ \\
\hline Exacerbation frequency (times/year) & $0.51 \pm 0.27$ \\
\hline
\end{tabular}

$\mathrm{BMI}$ - body mass index; ${ }^{*}$ includes only 28 current/ex-smoker. Values are mean \pm standard deviation (SD) or median (range).

\section{Statistical analysis}

Data were analyzed using the Statistical Package for Social Sciences (SPSS), version 18.0. Normality of distribution was detected with Kolmogorov-Smirnov test. Normally distributed data were expressed as mean \pm standard deviation (SD), while the non-normally distributed data were presented as median (range). The differences of normally distributed data between acute exacerbation and convalescence phase of COPD were statistically compared using an paired Student's $t$-test; The non-normally distributed data were analyzed using Wilcoxon signed ranks test. Correlation between changes of Gal-3 and hsCRP was examined using Pearson's correlation analysis. The differences of Gal-3 among groups (never smokers, current smokers, and ex-smokers) of COPD were examined using Mann-Whitney $U$ test. The associations between the serum Gal-3 level and COPD gender, age, smoking history, course of disease, $\mathrm{FEV}_{1} \%$ pred were determined to use multiple linear regression analysis. The level of statistically significant difference was set at $p<0.05$.

\section{Results}

\section{Patient characteristics}

Table 1 summarizes the demographic characteristics of all patients. A total of 44 patients were evaluated (34 males, $10 \mathrm{fe}$ males; age $69.18 \pm 9.36$ years). A total of 28 patients were current or former smokers ( 9 current smokers, 19 former smokers), they are mostly male, with a consumption of $21.4 \pm 14.4$ pack/ years. There were 16 patients who were never smokers who were exposed to high levels of air pollution, indoor pollution 
Table 2. Laboratory parameters of subjects.

\begin{tabular}{|cccc}
\hline Parameter & Acute exacerbation (range) & Convalescence (range) & P value \\
\hline White blood counts $(10 \% / L)$ & $9.54 \pm 3.46$ & $6.62 \pm 2.85$ & $<0.01$ \\
\hline Neutrophils $(\%)$ & $75.60 \pm 11.61$ & $60.80 \pm 6.51$ & $<0.01$ \\
\hline $\mathrm{PaO}_{2}((\mathrm{mmHg})$ & $62.22 \pm 13.14$ & $76.60 \pm 9.76$ & $<0.01$ \\
\hline $\mathrm{FEV}_{1} \%$ pred $(\%)$ & $\mathrm{ND}$ & $45.20 \pm 17.56$ & $\mathrm{ND}$ \\
\hline $\mathrm{FEV} / \mathrm{FVC}(\%)$ & $\mathrm{ND}$ & $44.43 \pm 11.63$ & $\mathrm{ND}$ \\
\hline $\mathrm{RV} / \mathrm{TLC}(\%)$ & $\mathrm{ND}$ & $46.0 \pm 8.3$ & $\mathrm{ND}$ \\
\hline Serum hsCRP $(\mathrm{mg} / \mathrm{dL})$ & $2.85 \pm 2.04$ & $0.58 \pm 0.37$ & $<0.01$ \\
\hline Serum Pro-BNP $(\mathrm{pg} / \mathrm{mL})$ & $1096.70 \pm 802.92$ & $\mathrm{ND}$ & $\mathrm{ND}$ \\
\hline Serum Galectin-3 $(\mathrm{ng} / \mathrm{mL})$ & $32.10 \pm 9.83$ & $29.02 \pm 8.68$ & $<0.01$ \\
\hline
\end{tabular}

$\mathrm{PaO}_{2}$ - arterial oxygen pressure; $\mathrm{FEV}_{1}$, forced expiratory volume at $1 \mathrm{~s} ; \%$ pred - percent of predicted value; FVC - forced vital capacity; RV - residual volume; TLC - total lung capacity; hsCRP - high-sensitivity C-reactive protein; ND - not done; Values are mean \pm standard deviation (SD) or median (range).
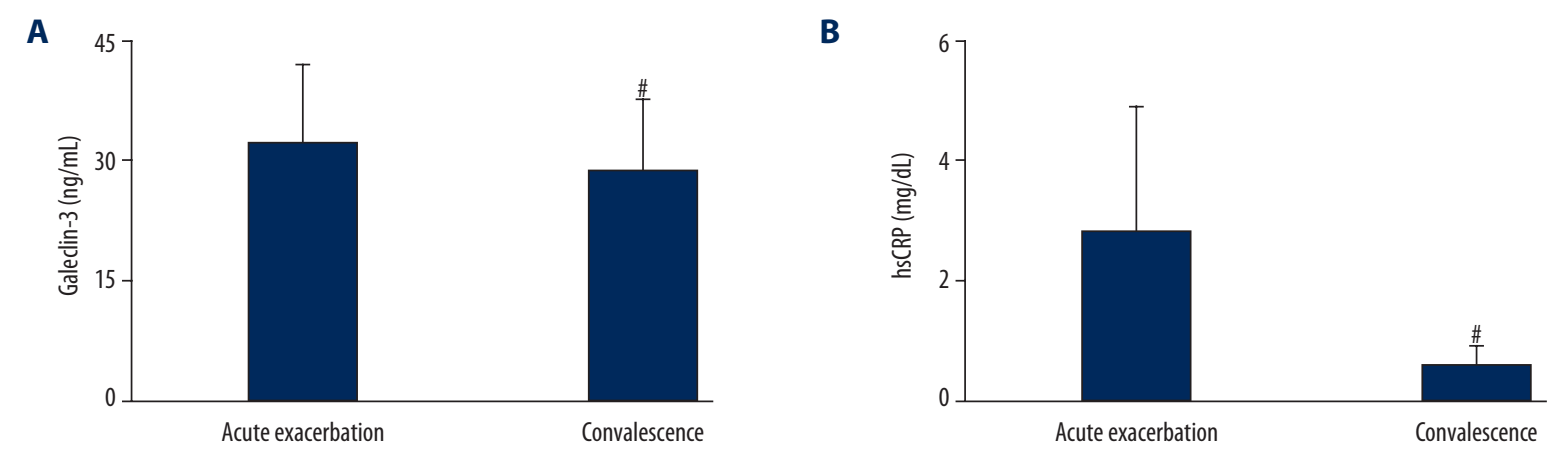

Figure 1. Analysis of concentrations of serum Gal-3 (A) and hsCRP (B) between acute exacerbation and convalescence of COPD ( $n=44)$. Values of Gal-3 were expressed as mean + SD and values of hsCRP were expressed as medians (interquartile range). hsCRP - high-sensitivity C-reactive protein; COPD - chronic obstructive pulmonary disease; ${ }^{*} p<0.01$; SD, standard deviation.

from biomass cooking and heating, and occupational exposures (including organic and inorganic dusts) from their declarations. The hospital days for AECOPD were 12 (range 10-14) days, and the frequency of acute exacerbation in the previous three years was $0.51 \pm 0.27$ times/year.

\section{Laboratory measurements}

Total leucocytes counts, neutrophils\%, arterial blood gas analysis, and hsCRP were recorded at the time of admission and discharge. Owing to worsening of physical status, the pulmonary function was not performed at the time of admission and was examined before patient discharge. The laboratory parameters of patients are shown in Table 2.

\section{Serum Gal-3 and hsCRP levels in acute exacerbation and convalescence phase of COPD}

The changes of serum Gal-3 and hsCRP levels are shown in Table 2 and Figure 1A, 1B. There was a significant decline in serum Gal-3 $(p<0.01)$ and hsCRP $(p<0.01)$ levels from acute exacerbation to convalescence phase in COPD.

\section{Correlation of the changes of Gal-3 and hsCRP/pro-BNP in acute exacerbation of COPD}

The correlation between the changes of Gal-3 level and hsCRP level in acute exacerbation of COPD is shown in Figure 2, and the correlation between the changes of Gal-3 level and proBNP level in acute exacerbation of COPD is shown in Figure 3. The analysis indicated a positive correlation between the levels of Gal-3 and hsCRP ( $r=0.354, p=0.018)$ in AECOPD, and the 


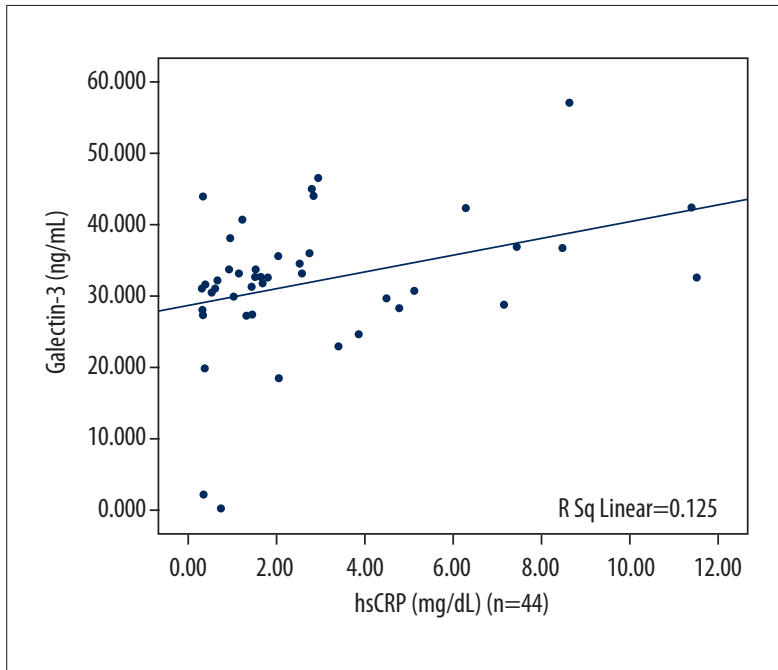

Figure 2. The correlation of the changes of Gal-3 and hsCRP in exacerbation phase in COPD $(n=44, r=0.354, p=0.018)$.

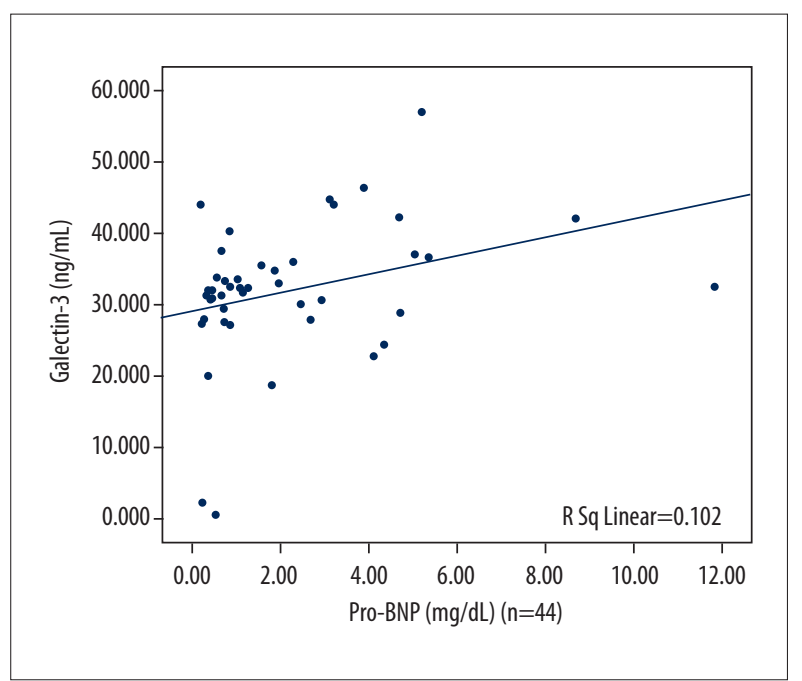

Figure 3. The correlation between the changes of Gal-3 and pro-BNP levels in acute exacerbation in COPD $(n=44$, $r=0.319, p=0.035$ ).
Table 3. Correlation of the level of Gal-3 in convalescence phase of COPD and the degree of airflow obstruction*.

\begin{tabular}{ccc} 
& $\begin{array}{c}\text { Gal-3 levels of convalescence of } \\
\text { COPD }(\mathbf{n g} / \mathbf{m L})\end{array}$ \\
\hline Parameter & $\mathbf{r}_{\mathrm{s}}$ & $\boldsymbol{P}$ \\
\hline $\mathrm{FEV}_{1} / \mathrm{FVC}(\%)$ & 0.266 & 0.155 \\
\hline $\mathrm{FEV}_{1} \%$ pred $(\%)$ & 0.122 & 0.453 \\
\hline
\end{tabular}

* Spearman's rank order method. $\mathrm{FEV}_{1}$ - forced expiratory volume at $1 \mathrm{~s}$; FVC - forced vital capacity; \% pred - percent of predicted value.

same result was observed between the changes of Gal-3 and pro-BNP ( $r=0.319, p=0.035)$ in AECOPD.

\section{Correlation of the level of Gal-3 in convalescence phase of COPD and the degree of airflow obstruction}

The associations between serum Gal-3 in the convalescence phase of COPD and $\mathrm{FEV}_{1} / \mathrm{FVC}$ or the $\mathrm{FEV}_{1} \%$ pred in the convalescence phase of COPD is shown in Table 3. The results showed that the Gal-3 level was not dramatically correlated with either of these parameters of lung function.

\section{Correlation between the level of Gal-3 and baseline characteristics in COPD}

In the multivariate linear regression analysis with Gal-3 as a dependent variable, COPD smoking status was found to be the only major influence factor affecting Gal-3 levels, whereas gender, age, course of disease, and $\mathrm{FEV}_{1} \%$ pred did not appear to be significant factors influencing Gal-3 levels (Table 4).

Table 4. Correlation between the level of Gal-3 and baseline characteristics in COPD.

\begin{tabular}{|c|c|c|c|}
\hline \multirow{2}{*}{ Parameter } & \multicolumn{3}{|c|}{ Multivariate linear regression analysis } \\
\hline & $\beta$ & $95 \% \mathrm{Cl}$ & $P$ value \\
\hline Sex (Male/Female) & 0.318 & $0.30-0.75$ & 0.752 \\
\hline Age (years) & 0.364 & $0.21-1.31$ & 0.161 \\
\hline Course of disease (years) & 0.872 & $0.84-3.60$ & 0.090 \\
\hline Smoking status & 0.458 & $1.02-1.09$ & 0.008 \\
\hline $\mathrm{FEV}_{1} \%$ pred $(\%)$ & 0.362 & $0.18-0.97$ & 0.215 \\
\hline
\end{tabular}

$\mathrm{FEV}_{1}$ - forced expiratory volume at $1 \mathrm{~s} ; \%$ pred - percent of predicted value; $\mathrm{P}<0.05$ was considered significant.

\section{6}

Indexed in: [Current Contents/Clinical Medicine] [SCI Expanded] [ISI Alerting System] [ISI Journals Master List] [Index Medicus/MEDLINE] [EMBASE/Excerpta Medica] [Chemical Abstracts/CAS] [Index Copernicus] 
Table 5. Correlation between the level of Gal-3 and smoking status in COPD.

\begin{tabular}{|cccccccc}
\hline Parameter & Never smokers & Ex-smokers & Current smokers & P1 & P2 & P3 \\
\hline Age $($ year) & $68.69 \pm 10.77$ & $71.84 \pm 7.24$ & $64.44 \pm 9.62$ & 0.315 & 0.272 & 0.052 \\
\hline BMI $\left(\mathrm{kg} / \mathrm{m}^{2}\right)$ & $22.66 \pm 3.70$ & $22.62 \pm 3.04$ & $21.07 \pm 2.21$ & 0.967 & 0.236 & 0.236 \\
\hline FEV $\%$ pred $(\%)$ & $46.07 \pm 17.17$ & $43.54 \pm 15.66$ & $47.14 \pm 23.29$ & 0.680 & 0.886 & 0.622 \\
\hline FEV $/$ FVC $(\%)$ & $43.32 \pm 10.91$ & $45.09 \pm 13.66$ & $45.00 \pm 9.04$ & 0.663 & 0.736 & 0.985 \\
\hline Galectin-3 $(\mathrm{ng} / \mathrm{mL})$ & & & & 0.036 & 0.388 \\
\hline Acute exacerbation & $29.12 \pm 11.73$ & $30.39 \pm 10.25$ & $33.91 \pm 3.55$ & 0.424 & 0.036 \\
\hline Convalescence & $27.76 \pm 9.68$ & $28.44 \pm 9.64$ & $30.94 \pm 3.40$ & 0.578 & 0.045 & 0.378 \\
\hline
\end{tabular}

$\mathrm{FEV}_{1}$ - forced expiratory volume at $1 \mathrm{~s}$; \% pred - percent of predicted value; $P 1$ - ex-smokers compared with never smoker; $P 2$ - current smokers compared with never smoker; $P 3$ - current smokers compared with ex-smokers.

\section{Correlation between the level of Gal-3 and smoking status in COPD}

Patients with COPD were classified into three groups according to smoking history: never smokers, current smokers and ex-smokers. Table 5 shows that the level of Gal-3 was the highest in the current smokers group, and the lowest in never-smokers group in either acute exacerbation or convalescence phase of COPD.

\section{Discussion}

In this study, we examined the Gal-3 levels in different phases of COPD and analyzed the potential correlations of circulating Gal-3 with the degree of airflow obstruction and the established biomarkers, including pro-BNP and hsCRP. The main findings of this study are as follows. First, serum Gal-3 levels increased in the acute exacerbation phase compared to the convalescence phase of COPD. Second, in the analysis of stratification, Gal-3 levels were higher in current-smokers than never-smokers, indicating that the circulating Gal-3 level may have a relationship with smoking history; and different mechanisms may exist between smokers and non-smokers among COPD patients. Third, Gal-3 level had a positive correlation with the pro-BNP and hsCRP levels in AECOPD.

COPD is accompanied by systemic inflammation that occurs as a result of many mechanisms, particularly airway inflammation with increasing numbers of specific inflammatory cell types, including macrophages, neutrophils, and lymphocytes in different parts of the lung [2,14-16]. These cells release inflammatory mediators and enzymes, and then induce structural alterations of airways and vasculature [14]. The pathological changes of COPD include destruction of the respiratory bronchioles, fibrosis of terminal and respiratory bronchioles, goblet cell and squamous metaplasia, and intimal thickening with smooth muscle proliferation $[1,16]$. In general, the inflammatory and structural changes in the airways lead to frequent exacerbations and comorbidities, which aggravate the overall severity in COPD patients and make it difficult to effectively administer current therapies [15,17]. In COPD, airway inflammation, which is mainly caused by cigarette smoke through inducing airway epithelial injury and increasing susceptibility to respiratory pathogens, plays a pivotal role in airway fibrosis $[17,18]$. Evidence indicates that Gal-3 is a mediator of profibrotic pathways, induces fibroblast to proliferation and transformation, and mediates collagen deposition $[3,4,19]$. Moreover, Gal-3 is involved in many processes, including cell adhesion, proliferation, apoptosis, and cytokine secretion [3,20]. Gal-3 may exert an important regulatory effect on the pathogenesis of COPD, as recent findings have revealed that elevated Gal-3 expression correlates with increased epithelial injury and airflow obstruction in severe COPD patients [13,21,22]. Lucia et al. [23] reported an enhanced blood Gal-3 level in COPD patients compared with healthy controls. In addition, Simon et al. [22] demonstrated that Gal-3 is upregulated in lung tissues of patients with emphysema when compared with healthy volunteers. They have also discovered that rats with cigarette smoking expose, overexpressing Gal-3 can induce airway inflammation compared with non-susceptible rats $[22,24]$. The elevated level of Gal-3 has also been found in airway epithelia cells (AECs) from COPD patients, as well as in lung fluid or serum from COPD patients compared to smoking and nonsmoking controls [24]. It has also been reported that Gal-3 increases the secretion of neutrophil attractant CXCL8 in AECs and induces a pro-inflammatory response in AECs from COPD patients [22]. In summary, these data indicate that Gal-3 may have a causal relationship with COPD.

Our study indicated that serum Gal-3 levels in the exacerbation phase of COPD was higher than that in the convalescence phase of COPD, which suggests that serum levels of Gal-3 correlate with COPD exacerbation attacks. HsCRP increases during bacterial infections, which could be the trigger for exacerbations 
in patients with COPD. HsCRP level is used as an inflammatory biomarker in evaluating COPD exacerbation [14]. We found that serum Gal-3 levels and hsCRP levels both were increased in AECOPD patients. Moreover, serum Gal-3 levels were positively correlated with serum CRP levels. These findings suggest that Gal-3 exerts a potential effect on the pathogenesis of inflammation of COPD and may serve as a specific inflammatory biomarker at the site of tissue inflammation as a supplement to conventional acute phase protein. Pro-BNP is an established serum marker for the diagnosis and prognosis in heart failure caused by COPD $[6,7,25]$. It has been reported that pro-BNP levels are modestly higher during exacerbations than in stable patients [25-27]. We also found that serum Gal-3 levels correlated positively with pro-BNP, consistent with a previous study reported by Lucia [23], suggesting that Gal-3 may have been involved in the process of right ventricle dysfunction.

There were some limitations to this study that need to be considered. First, the sample size was relatively small and participants were recruited only at a single center. Future studies

\section{References:}

1. Global Strategy for the Diagnosis, Management and Prevention of COPD, Global Initiative for Chronic Obstructive Lung Disease (GOLD) 2015. http:// www.goldcopd.com

2. MacNee W: Systemic inflammatory biomarkers and co-morbidities of chronic obstructive pulmonary disease. Ann Med, 2013; 45(3): 291-300

3. Calvier L, Miana M, Reboul P et al: Galectin-3 mediates aldosterone-induced vascular fibrosis. Arterioscler Thromb Vasc Biol, 2013; 33(1): 67-75

4. Numano F, Shimizu C, Jimenez-Fernandez $S$ et al: Galectin-3 is a marker of myocardial and vascular fibrosis in Kawasaki disease patients with giant aneurysms. Int J Cardiol, 2015; 201: 429-37

5. Uluca Ü, Şen V, Ece A et al: Serum galectin-3 levels in children with chronic hepatitis B infection and inactive hepatitis B carriers. Med Sci Monit, 2015; 21: $1376-80$

6. Batlle M, Campos B, Farrero $M$ et al: Data on clinical characteristics of a heart failure patients' cohort with reduced ejection fraction and analysis of the circulating values of five different heart failure biomarkers; high sensitivity troponin T, galectin-3, C-terminal propeptide of type I procollagen, soluble AXL and BNP. Data Brief, 2016; 9: 876-82

7. Bosnjak I, Selthofer-Relatic K, Vcev A: Prognostic value of galectin-3 in patients with heart failure. Dis Markers, 2015; 2015: 690205

8. Kosacka M, Piesiak P, Kowal A et al: Galectin-3 and cyclin D1 expression in non-small cell lung cancer. J Exp Clin Cancer Res, 2011; 30: 101

9. Gao P, Gibson PG, Baines KJ et al: Anti-inflammatory deficiencies in neutrophilic asthma: Reduced galectin-3 and IL-1RA/IL-1beta. Respir Res, 2015; 16: 5

10. Mackinnon AC, Gibbons MA, Farnworth SL et al: Regulation of transforming growth factor-beta1-driven lung fibrosis by galectin-3. Am J Respir Crit Care Med, 2012; 185(5): 537-46

11. Honig E, Schneider K, Jacob R: Recycling of galectin-3 in epithelial cells. Eur J Cell Biol, 2015; 94(7-9): 309-15

12. Nakajima K, Kho DH, Yanagawa T et al: Galectin-3 in bone tumor microenvironment: A beacon for individual skeletal metastasis management. Cancer Metastasis Rev, 2016; 35(2): 333-46

13. Pilette $\mathrm{C}$, Colinet B, Kiss R et al: Increased galectin-3 expression and intraepithelial neutrophils in small airways in severe COPD. Eur Respir J, 2007; 29(5): 914-22

14. Koutsokera A, Kiropoulos TS, Nikoulis DJ et al: Clinical, functional and biochemical changes during recovery from COPD exacerbations. Respir Med 2009; 103(6): 919-26 with larger sample sizes should be performed to confirm these results. Second, our study was not designed to explore the mechanistic pathways leading to higher Gal-3 levels in patients with AECOPD. Despite such limitations, this study provides preliminary information about changes in Gal-3 levels in the COPD exacerbation phase.

\section{Conclusions}

The present study shows that the levels of serum Gal-3 and hsCRP were elevated in AECOPD and tended to decline in the convalescence phase of COPD; the level of Gal-3 correlated with hsCRP and Pro-BNP levels. Meanwhile, the level of serum Gal-3 in patients with COPD was influenced by smoking status. Our findings suggested that Gal-3 might be a valuable biomarker for AECOPD. Further studies are needed to determine if this biomarker has a causal role in the disease and has correlations with different COPD phenotypes.

15. Berg K, Wright LL: The pathology of chronic obstructive pulmonary disease: Progress in the $20^{\text {th }}$ and $21^{\text {st }}$ centuries. Arch Pathol Lab Med, 2016; 140(12): 1423-28

16. Rahman : Oxidative stress in pathogenesis of chronic obstructive pulmonary disease: Cellular and molecular mechanisms. Cell Biochem Biophys, 2005; 43(1): 167-88

17. Yanagisawa $\mathrm{H}$, Hashimoto $M$, Minagawa $S$ et al: Role of IL-17A in murine models of COPD airway disease. Am J Physiol Lung Cell Mol Physiol, 2017; 312(1): L122-30

18. Du Y, Xue Y, Xiao W: Association of IREB2 gene rs2568494 polymorphism with risk of chronic obstructive pulmonary disease: A meta-analysis. Med Sci Monit, 2016; 22: 177-82

19. Koca SS, Akbas F, Ozgen $M$ et al: Serum galectin-3 level in systemic sclerosis. Clin Rheumatol, 2014; 33(2): 215-20

20. Cheng D, Liang B, Li Y: Serum galectin-3 as a potential marker for gastric cancer. Med Sci Monit, 2015; 21: 755-60

21. Mukaro VR, Bylund J, Hodge $G$ et al: Lectins offer new perspectives in the development of macrophage-targeted therapies for COPD/emphysema. PLoS One, 2013; 8(2): e56147

22. Pouwels SD, Hesse L, Faiz A et al: Susceptibility for cigarette smoke-induced DAMP release and DAMP-induced inflammation in COPD. Am J Physiol Lung Cell Mol Physiol, 2016; 311(5): L881-92

23. Agoston-Coldea L, Lupu S, Petrovai D et al: Correlations between echocardiographic parameters of right ventricular dysfunction and Galectin-3 in patients with chronic obstructive pulmonary disease and pulmonary hypertension. Med Ultrason, 2015; 17(4): 487-95

24. Heijink IH, Pouwels SD, Leijendekker C et al: Cigarette smoke-induced damage-associated molecular pattern release from necrotic neutrophils triggers proinflammatory mediator release. Am J Respir Cell Mol Biol, 2015; 52(5): 554-62

25. Hawkins NM, Khosla A, Virani SA et al: B-type natriuretic peptides in chronic obstructive pulmonary disease: A systematic review. BMC Pulm Med, 2017; 17(1): 11

26. Høiseth AD, Omland T, Hagve TA et al: NT-proBNP independently predicts long term mortality after acute exacerbation of COPD - a prospective cohort study. Respir Res, 2012; 13: 97

27. Buchan A, Bennett R, Coad A et al: The role of cardiac biomarkers for predicting left ventricular dysfunction and cardiovascular mortality in acute exacerbations of COPD. Open Heart, 2015; 2(1): e000052 